\title{
The microbiological diagnosis of Chlamydia trachomatis infections: milestones from a centenary history
}

\author{
Enrico Magliano
}

Scientific Director, Italian Association of Clinical Microbiologists, Milan, Italy

August Compte, a French philosopher of science, back in the 18th Century wrote: You do not know a science, if you do not know its history.

It is ambitious to adapt this aphorism to the history of the microbiological diagnosis of Chlamydia trachomatis infection, but the father of positivism would probably forgive me...

In 1907, in Java, Ludwig Halberstaeder and Stanislaus von Prowazec described - using a light microscope - the presence of intracytoplasmatic inclusions in samples of conjunctival scrapings obtained from an orangutan that was experimentally infected with ocular material from a patient suffering from trachoma.

They made this observation by using the differential staining method patented three years before by Gustav Giemsa (10).

These scientists described not only the large intracytoplasmatic inclusions, but also some small extracellular particles. This was the first time that these microorganisms were classified as Chlamidozoa. It is well know that these microbes were for a long time considered as viruses.

Nowadays, Chlamydia spp. are classified as obligate intracellular bacteria that must parasitize eukaryotic cells to achieve a full developmental cycle. $C$. trachomatis shows a particular tropism for the genital and conjunctival epithelia and encompasses 19 different serovars, which are predominantly pathogenic for the urogenital tract (6). Correspondence: Enrico Magliano, AMCLI, Via Farini 81, 20159 Milano (MI).
E-mail: e.magliano@libero.it

Key words: Chlamydia trachomatis infection; history.

Conflict of interest: the authors declare no potential conflict of interest.

Received for publication: 21 June 2016.

Accepted for publication: 21 June 2016.

(c) Copyright E. Magliano, 2016

Licensee PAGEPress, Italy

Microbiologia Medica 2016; 31:6103

doi: $10.4081 / \mathrm{mm} .2016 .6103$

This article is distributed under the terms of the Creative Commons Attribution Noncommercial License (by-nc 4.0) which permits any noncommercial use, distribution, and reproduction in any medium, provided the original author(s) and source are credited.

\section{Staining methods}

Staining Chlamydiae with the Gram method gives extremely poor results for two main reasons: i) these microbes are growing in an intracellular site and ii) any muramic acid is lacking in their cell wall.

Although the direct detection of Chlamydia in appropriate cytological smears was performed by Gupta in 1979 (9), the diagnostic value of the method based on direct smear was questioned and not approved (15).

As an alternative technique, the iodine stain is much simpler for the detection of Chlamydia related intracytoplasmatic inclusions in infected cells, based on the large production of iodine stainable glycogen by $C$. trachomatis (19).

\section{Culture}

Back in 1932, the so-called lymphogranuloma venereum (LGV) agent was first cultivated in Guinea pigs' kidney and testicles (16). The culture of clinical samples on cells monolayer (largely used the McCoy line, derived from mouse connective tissue) was for a longtime the gold standard (8) in Chlamydia diagnostics. The characteristic chlamydial inclusion body can also (and likely with a higher specificity) be detected after staining with fluorescence conjugated specific (either polyclonal but preferably monoclonal) antibodies that bind to different targets, including the chlamydial major membrane protein (MOMP) or the LPS. The sensitivity of culture-based techniques varies from $50 \%$ to $90 \%$ (3) depending on selected settings.

Culture is nowadays considered a slow, labour-intensive and hence quite costly method and it is very seldom used for routine diagnostics. Nevertheless, it retains an excellent level of specificity and allows the in vitro antibiotic susceptibility testing and the determination of serovar specificity of isolated strains, which may be advantageous in epidemiological studies.

At the end of the 1980s, two new diagnostic approaches become available for the detection of Chlamydia spp. specific antigens in the clinical microbiology laboratory: the enzyme linked immunoassays (EIA) and the direct detection by immunofluorescence (DFA).

\section{Enzyme linked immunoassays}

This method allows the direct antigen detection based on the well-known enzyme-linked immunoassay technique (12). The average sensitivity of EIAs is lower, compared with the cell culture procedure, but their negative predictive value is high. The EIAbased methods can be performed in a single test format, but more 
frequently these techniques are fully automated with a softwarebased interpretation of results.

In Italy, in 1988, an EIA-based national wide-large epidemiological study about the urogenital Chlamydia infections in patients attending specialized department of Roma, Milano and Torino was performed and this is still one of the most comprehensive study ever done in Italy in the field of sexually transmitted infections (STI) (24).

\section{Direct detection by immunofluorescence}

The direct fluorescent antibody test has high sensitivity in particular when cervical specimens are tested.

The method is somehow complicated since the requirement of highly trained and specialized operators is required in order to keep high level of clinical performance and it is not suitable for processing large numbers of sample (17) simultaneously.

\section{Serology based techniques}

Until 1936, the complement fixation test, devised by S.B Bedson, Professor of Bacteriology at the London Hospital (1), was the only available method for the diagnostic detection of the antibody response. Among serological techniques, the Bedsonian complement fixation test has been a time-honoured method, but subsequently it was shown to have limited clinical diagnostic value. When applied to the issue of Chlamydia infections, a cross reactivity was clearly observed among cases of psittacosis and oculo genital infections, likely due to the wide cross reactivity of the antigens used (2). In 1962, Nichols and Mc Comb suggested the use of fluorescent-labelled antibodies method for the serological differentiation of Chlamydia spp. (18).

Later on, in 1970, Wang and Grayston (26) developed a new indirect micro-titre immunofluorescence typing test, which confirmed the existence of six major chlamydia serotypes involved in oculogenital infections. In 1975, the same Authors identified an additional group of 15 more serotypes. In 1983, Saikku e Paavonen (22) developed the first solid-phase enzyme assay (EIA) for detection of Chlamydia specific antibodies.

The use of serological test for diagnosis of uncomplicated $C$. trachomatis infections, due to the low sensitivity and specificity of the method has a very limited clinical value. As the 2016 European Guideline for the management of NGU suggest (13), the serologic data have only a limited clinical value and could be, if interpreted with caution, of some aid in the diagnosis of PID, ectopic pregnancy, reactive arthritis, neonatal pneumonia and tubal infertility.

\section{Nucleic acid amplification test}

The first experimental molecular characterization of Chlamydia were performed at the end of the last century's 80 s (20) followed by the development of many different molecular diagnostic based methods for the practical clinical use (23).

Actually, the diagnostic reference methods are based on NAATs (Nucleic Acid Amplification Test) technology that has intrinsic high specificity and sensitivity. By using NAATs the detectability of chlamydial infections has increased by some $50 \%$ compared to the previous methods (25).
Moreover, by using NAATs, it is possible to obtain the diagnosis with the usage of self-collectable and non-invasively obtained clinical samples such as first void urine or self-taken vaginal swabs. Today only NAATs are recommended by National and International Reference Centres and by international Guide lines $(5,13)$.

The POC (Point of Care) tests commonly based on antigen membrane capture on lateral flow assays are simple to perform, but these methods have low and clinically not adequate sensitivity so that their overall performance is alarming poor (14).

The Clinical Microbiologist, who participates to the on-going revolutionary evolution of the diagnostics tools, must be, at the same time, well informed and aware about the social and behaviour modifications that have developed since the last century. In particular, it is of fundamental relevance to have a deep knowledge of all the novel sexual risk behaviours, especially among the most vulnerable communities, that have generated an increased risk for the acquisition and spreading of STI. Only being a major player in the game, the Clinical Microbiologists could be effective in the implementation of effective preventive measures.

The recent Editorial published in the British Medical Journal (4) on the Chem sex underlined that violent, traumatic prolonged (days) sexual intercourse may facilitate the bacterial invasion of the GU tract. Again, recently, the Lymphogranuloma venereum has been reported as a re-appearing infection in vulnerable drug addicted patients in selected communities. It is so highly probable that the facilitated bacterial invasion is increased following violent and prolonged intercourse: and this will be a major issue also in Italy.

The 2016 Report of the EDC on the guidance for the control of Chlamydia infection in Europe (7 march 2016) confirmed that Chlamydia related disease are the most frequently reported sexual transmitted infection in the EU member States and in the neighbouring Countries (11). As a consequence, the Public Health System in England published in March 2016 a Report of the National Chlamydia Screening Program (21). This report has generated a set of estimates about the epidemiology of chlamydia infections, from its incidence, prevalence and duration of infection, and its role in the pathogenesis of PID and tubal factor infertility (TFI). This report clearly supported the necessity to implement a nationwide screening of prevalent cases to prevent PID cases and suggested that greater emphasis should be placed on the detection and treatment of incident $C$. trachomatis infections.

Data have also been confirmed in our country by a collaborative joint effort between the Istituto Superiore di Sanità (Centro Operativo AIDS) and the AMCLI Study group on STI (GLIST).

Another stimulating field of interest are the studies performed on pathogenesis of the genital tract diseases due to $C$. trachomatis (7). Although the pathologic consequences of $C$. trachomatis genital infections are well established, the mechanisms that result in the chlamydia induced tissue damage are not fully understood. Abundant in vitro data suggest that inflammatory response to chlamydia is initiated and sustained by actively infected nonimmune host epithelial cells. Continued studies about the complex molecular and cellular interactions between these pathogen and the complex system of the human GU tract are needed to address the still relevant gaps in our understanding of these mechanisms.

At the end of this quite concise synthesis of the long history of the relationship between the clinical microbiologists and some of the most intriguing germs, such as chlamydiae, it is obvious to me to recall when during the International Meeting on Chlamydia that AMCLI organized in Como (21-22 may 2009) I asked my Colleague David Taylor Robinson, one of the most expert and prominent scien- 
tist that dedicated almost his entire life to the study of STI: Which is the role of the Clinical Microbiologist in the investigation of chlamydia related infections?. Well, his trenchant answer was Just fundamental.

\section{References}

1. Bedson SP, Western GT, Simpson SL. Observation of the etiology of psittacosis. Lancet 1930;1:235.

2. Bedson SP. Immunological studies with the virus of Psittacosis. Br J Exp Pathol 1933;14:3.

3. Blach CM. Current methods of laboratory diagnosis of Chlamydia trachomatis infection. Clin Microbiol Rev 1996;1: 160-84.

4. McCall H, Adams N, Mason D, Willis J4. What is chemsex and why does it matter? BMJ 2015;351.

5. CDC. Recommendation for the laboratory based detection of Chlamydia trachomatis and Neisseria gonorrhoeae. 2014. Available from: http://www.cdc.gov/mmwr/preview/mmwrhtml/ rr6302a1.htm

6. Cevenini R, Donati M, Sambri V. Chlamydia trachomatis: the agent. Best Pract Res Clin Obstet Gynaecol 2002;16:761-73.

7. Darville T, Hiltke TY. Pathogenesis of genital tract disease due to Chlamydia trachomatis. J Infect Dis 2010;201:2-18.

8. Gordon FB, Quan AL. Isolation of the trachoma agent in cell culture. Proc Soc Exp Biol Med 1965;118:354-9.

9. Gupta P, Lee Y, Erozan YS, et al. Cytologic investigations in Chlamydia infection. Acta Cytologica 1979;23:315-20.

10. Halbers Taedter L, Von Provazec S. Uber Zelleinschlüsse parasitärer Natur beim Trachom. Arbeiten aus dem Kaiserlichen Gesundheitsamte, Berlin 1907;26:44-7.

11. How to control Chlamydia: an ECDC guidance for Europe. Available from: http://ecdc.europa.eu/en/publications/ Publications/chlamydia-control-europe-guidance.pdf (accessed on June 16th, 2016).

12. Howard LV, Coleman PF, England BJ, et al. Evaluation of chlamydiazyme for the detection of genital infections caused by Chlamydia trachomatis. J Clin Microbiol 1986;23:329-32.

13. Horner PJ, Blee K, Falk L, et al. 2016 European guideline on the management of non-gonococcal urethritis. Int J STD AIDS. 2016 May 4. [Epub ahead of print].

14. Jannie J, van der Helm, Sabajo LOA, et al. Point of care test for detection of urogenital Chlamydia in women shows low sensitivity. A performance evaluation study in two clinics in Suriname. PLoS One 2012;7:e32122.

15. Leopardi 0, Magliano E, Garlaschi C. Cytological, immunocytological and serological diagnosis of Chlamydia trachomatis cervicitis. Cervix 1983;1:45.

16. Meyer K, Anders HE, Versuche Zur Zuchtng des lymphogranuloma inguinale-virus. Berl Klin Woch 1932;11:318-21.

17. Nichols RL, Mc Comb D. Immunofluorescent studies with trachoma and related antigens. J Immunol 1962;89:545-54.

18. Nichols RL, Mc Combe DE. Serological strain differentiation in trachoma. J Exp Med 1964;120:639-45.

19. Orfila H, Boulanger JC. [Chlamydia trachomatis urogenital infections in women. Best diagnostic approaches]. Gynecol Obstet Fertil 2004;32:1064-74.

20. Pao CC, Lin SS, Yang TE, et al. Deoxyribonucleic acid hybridization analysis for the detection of urogenital Chlamydia trachomatis infections in women. Am J Obstet Gynecol 1987;156:195-9.

21. Price MJ, Ades AE, Soldan K, et al. The natural history of Chlamydia trachomatis infection in women. A multiparameter evidence synthesis. Health Technol Assess 2016;20-2:1-243.

22. Saikku P, Paavonen Y, Vaannen P, et al. Solid-phase enzyme immunoassay for chlamydial antibodies. JCM 1983;17:22-7.

23. Schacter J, Chow JM, Howard H, et al. Detection of Chlamydia trachomatis by nucleic acid amplification testing. J Clin Microb 2006;44:2512-7.

24. Sessa R, Latino MA, Magliano E, et al. Epidemiology of urogenital infection caused by Chlamydia trachomatis and outline of characteristic features of patients at risk. J Med Microbiol 1994;3:168-72.

25. UK National Guideline for the management of Chlamydia trachomatis. 2015. Available from: http://www.bashh.org/documents/UK\%20guideline\%20for\%20the\%20management\%20of\% 20\%20Chlamydia\%20trachomatis\%20(8-06-15\%20v4)\%20submitted\%20to\%20IJSA.pdf

26. Wang SP, Grayston YT. Human serology in Chlamydia trachomatis infection with immunofluorescence. J Infect Dis 1974;130: 388-97. 